\title{
Association of antimicrobial resistance and gut microbiota composition in human and non-human primates at an urban ecotourism site
}

\author{
C. W. Chong ${ }^{1}$, A. H. S. Alkatheeri ${ }^{2}$, N. Ali ${ }^{3}$, Z. H. Tay ${ }^{3}$, Y. L. Lee ${ }^{3}$, S. J. Paramasivam ${ }^{4,7}$, K. Jeevaratnam ${ }^{4}$, W. Y. Low ${ }^{5,6}$ \\ and S. H. E. Lim², $3^{*}$
}

\begin{abstract}
Background: The rise of nature-based ecotourism in the past decade has introduced unprecedented challenges in managing the increasing interaction between humans and animals. The potential transmission of antibiotic resistant microbes between humans and non-human primate populations is a concern due to their genetic similarity. Malaysia is well known for hotspots of wild life diversity where non-human primates like monkeys and orangutans have become popular tourist attractions. In this study, we assessed the prevalence of antimicrobial resistant Staphylococcus aureus, Enterococcus species, and other Enterobacteriaceae in the faeces of human (HS) and two non-human primates (NHP) in Malaysia, the Long-tailed macaque (Macaca fascicularis, MF) and Silvered leaf monkey (Trachypithecus cristatus, TC). In addition, the faecal bacterial composition was profiled to evaluate the potential association between antibiotic resistant profiles and composition of gut microbiota.
\end{abstract}

Results: We tested the isolated bacteria using a selection of antibiotics. The results showed that both the number of antibiotic resistant strains and resistance level were higher in humans than NHPs. Overall, the composition of gut microbiome and pattern of antibiotic resistance showed that there was higher similarity between MF and TC, the two NHPs, than with HS. In addition, samples with higher levels of antibiotic resistance showed lower bacterial richness. Homo sapiens had the lowest bacterial diversity and yet it had higher abundance of Bacteroides. In contrast, NHPs displayed higher bacterial richness and greater prevalence of Firmicutes such as Ruminococceae and Oscillospira.

Conclusion: Higher antibiotic susceptibility in NHPs is likely related to low direct exposure to antibiotics. The lack of resistance may also suggest limited antimicrobial resistance transmission between humans and NHP. Nonetheless, continued monitoring over a long period will help mitigate the risk of anthropozoonosis and zooanthroponosis.

Keywords: Ecotourism, Non-human primates, Human animal interaction, Antibiotic resistance

*Correspondence: erinlimsh@gmail.com

2 Health Science Division, Abu Dhabi Women's College, Higher Colleges of Technology, 41012 Abu Dhabi, UAE

Full list of author information is available at the end of the article

\section{Background}

Ecotourism is one of the fastest growing sectors in Malaysia. Since the formulation of the National Ecotourism Plan, the number of tourists visiting Malaysia had soared from 5.5 million in 1998 to 27.5 million in 2015 [1]. As the bulk of tourists comprises international travelers, close human-animal interaction such 
as feeding may facilitate zooanthroponosis transfer, leading to mortality in the wild animal population [2]. This is exacerbated by the fact that tourists generally lack the fundamental knowledge on the risk of pathogen transmission to animals [3].

Ecotourism is a growing sector in many countries due to increasing appreciation and desire to observe and interact with animals in their natural setting. It provides for a more up-close and personal contact between tourists and wild animals whilst experiencing nature simultaneously which are considered more enjoyable [4]. Subsidiary benefits of ecotourism include support of conservation for natural ecosystems and the promotion of sustainable local development [5]. Nevertheless, the lack of awareness about pathogen transmission amongst tourists as well as non-controlled interactions among visitors and wildlife (due to the poor management by ecotourism operators) pose a potential healthcare risks such as transmission of infectious diseases [2]. Additionally, the close interaction may increase the transmission of antibiotic resistant strains between tourists and wild animals [6]. Separately, the transfer of non-pathogenic bacteria between human and animal may alter the composition of microbiome in their gut [7], and as a consequence, affects the ability of the host to resist colonization of exogenous bacteria [8]. Conversely, sick animals may increase the risk of zooanthroponosis, and transferring infections to tourists [9].

Further to the above, human animal interaction (HAI) plays a role in altering the behavioural routines of wild animals. For example, consistent feeding from tourists can results in wild animals spending less time foraging for food, socializing with each other and a reduction in travel distance $[10,11]$. Prolonged negative HAI is associated with more aggressive displays of behavior of animals towards tourists, culminating into cases of attack [12].

In this study, we focused on the non- human primates (NHP) from Kuala Selangor Nature Park as a nonconventional setting for transmission of antimicrobial resistance (AMR). We investigated NHPs in urban areas because they are known reservoir of zoonotic diseases to humans and are particularly popular at ecotourism destinations, especially within ASEAN. The aims of this study were to evaluate the prevalence of selected antibiotic-resistant bacteria in humans and in NHPs, in addition to understanding the relationship between gut bacterial composition and antibiotic resistant bacterial carriage rate of these hosts at an ecotourism site. To the best of our knowledge, this is the first report on antibiotic resistance and gut microbial composition of urban monkeys in Malaysia.

\section{Results}

Disk diffusion testing MRSA

A total of 61, 26 and 25 isolates were obtained from Macaca fascicularis (MF, Long-tailed macaque), Trachypithecus cristatus (TC, Silvered leaf monkey), and Homo sapiens (HS) respectively. The isolates were classified into resistant, intermediate and susceptible based on guidelines from Clinical and Laboratory Standards Institute (CLSI) (Table 1) and expressed in percentage (Fig. 1a). Based on ChromMRSA, all isolates obtained were $100 \%$ resistant to oxacillin. Resistance levels for cefoxitin were similar for MF and HS at 70\%, compared to TC at $40 \%$. No resistance was observed for linezolid and vancomycin across all hosts. Tetracycline was the most effective antibiotic for bacterial isolates obtained from MF and TC, achieving 100\% susceptibility. In comparison, only $25 \%$ of the isolates from HS were susceptible to tetracycline. Overall, a distinct antibiotic susceptibility pattern of MRSA across the three hosts was apparent (Fig. 2a).

\section{Enterococcus species}

A total of 108, 141 and 170 isolates were obtained from MF, TC, and HS, respectively. Around 70\% of the isolates from TC displayed resistance against penicillin while

Table 1 Antibiotics and amount impregnated per disk for MRSA, Enterococcus spp. and other Enterobacteriaceae

\begin{tabular}{lllll}
\hline Antibiotic & Disc potency $(\boldsymbol{\mu g})$ & \multicolumn{3}{l}{$\begin{array}{l}\text { Inhibition zone diameter } \\
(\mathbf{m m})\end{array}$} \\
\cline { 3 - 5 } & & $\mathbf{R}$ & $\mathbf{I}$ & $\mathbf{S}$ \\
\hline MRSA & & & & \\
Oxacillin & 1 & $\leq 17$ & - & $\geq 18$ \\
Tetracycline & 30 & $\leq 14$ & $15-18$ & $\geq 19$ \\
Linezolid & 30 & - & - & $\geq 21$ \\
Cefoxitin & 30 & $\leq 24$ & - & $\geq 25$ \\
Vancomycin & 30 & - & - & $\geq 15$ \\
Enterococcus spp. & & & & \\
Penicillin & 10 units & $\leq 14$ & - & $\geq 15$ \\
Ampicillin & 10 & $\leq 16$ & - & $\geq 17$ \\
Linezolid & 30 & $\leq 20$ & $21-22$ & $\geq 23$ \\
Tetracycline & 30 & $\leq 14$ & $15-18$ & $\geq 19$ \\
Vancomycin & 30 & $\leq 14$ & $15-16$ & $\geq 17$ \\
Other Enterobacteriaceae & 30 & & & \\
Cefazolin & 30 & $\leq 14$ & $15-17$ & $\geq 18$ \\
Ceftazidime & 30 & $\leq 14$ & $15-17$ & $\geq 18$ \\
Ampicillin & 10 & $\leq 13$ & $14-16$ & $\geq 17$ \\
Gentamicin & 10 & $\leq 12$ & $13-14$ & $\geq 15$ \\
Tetracycline & 30 & $\leq 11$ & $12-14$ & $\geq 15$ \\
\hline R & & & &
\end{tabular}

$R$ Resistant, I Intermediate, S Susceptible (CLSI, 2018) 


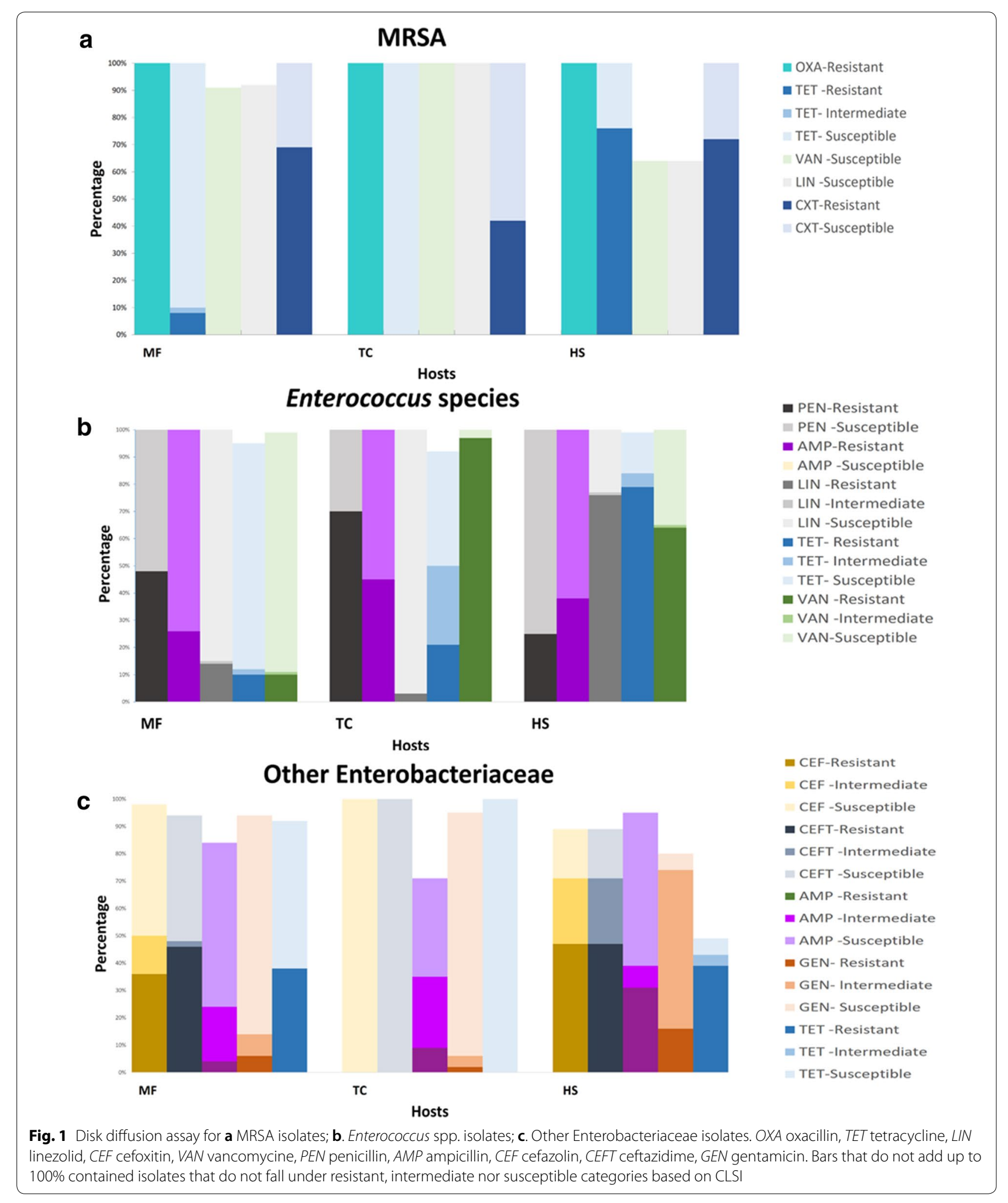



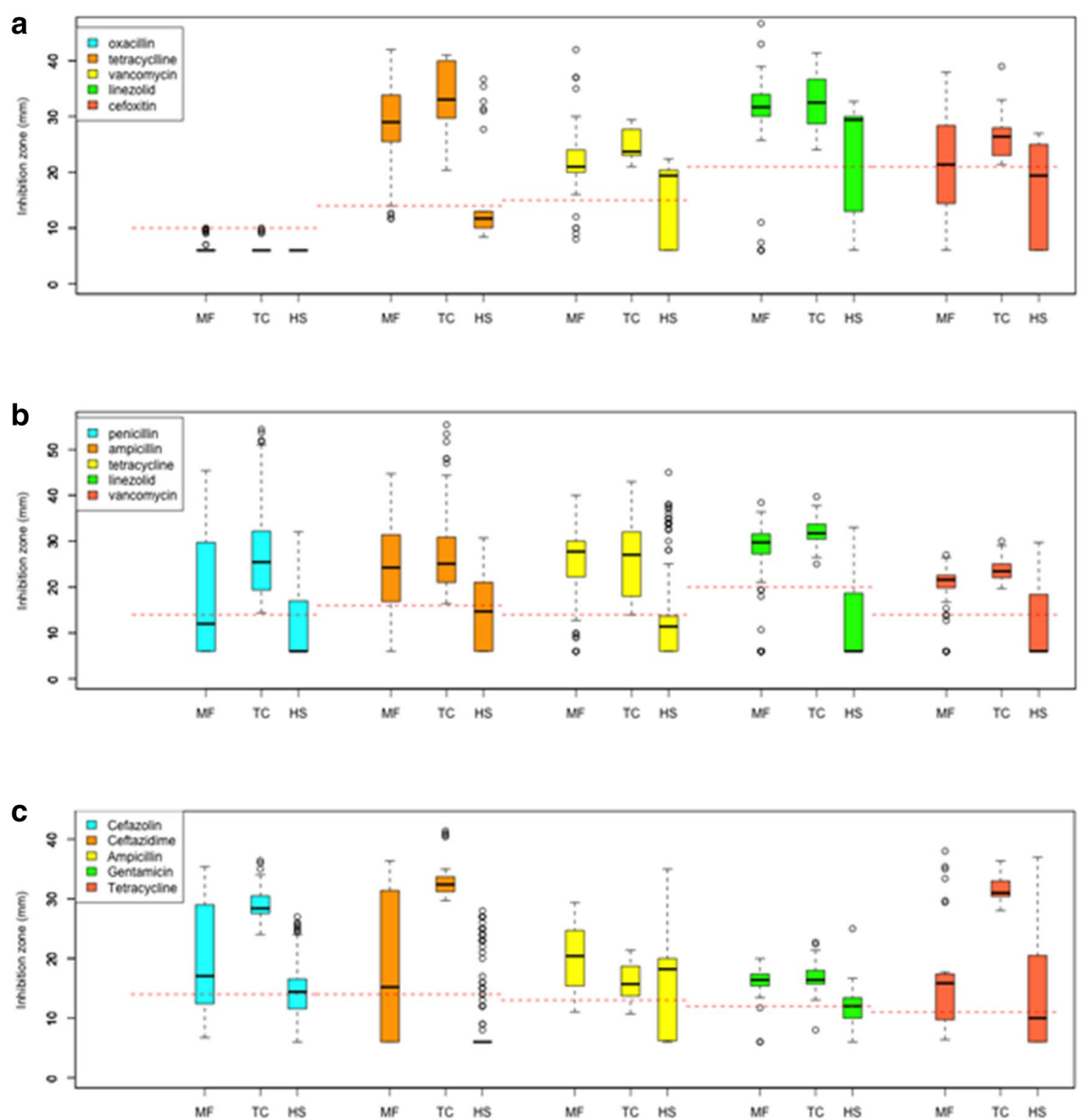

Fig. 2 Boxplot of antibiotic resistance profiles inferred based on inhibition zone (mm), a MRSA; b Enterococcus spp.; c other Enterobacteriaceae

approximately $50 \%$ of MF and $25 \%$ of $\mathrm{HS}$ isolates showed resistance. Resistance of MF to vancomycin was low at $10 \%$ compared to the TC at $97 \%$ and HS at $64 \%$ (Fig. 1b). HS isolates were $100 \%$ resistant to tetracycline (Fig. 2b). HS and TC isolates showed partial resistance to tetracycline. When all 5 antibiotics were compared, TC formed the tightest cluster amongst the three hosts (Fig. 3b).

\section{Other Enterobacteriaceae}

Fifty, 47 and 171 isolates were obtained for MF, TC, and HS respectively. Enterobacteriaceae from MF and HS were resistant to cefazolin and ceftazidime while
Enterobacteriaceae from TC showed 100\% susceptibility to both antibiotics (Fig. 1c).

\section{Comparison of fecal bacterial community composition between MF, TC and HS}

Significantly lower bacterial richness (Shannon's and Simpson's Diversity indices) and evenness were detected in HS in comparison to MF and TC (Additional file 1: Fig. S1). When the distribution of taxa was observed at the phyla level, higher similarity was apparent between MF and TC (the two NHPs) than with HS. For instance, the NHPs showed higher level of Firmicutes and lower level 

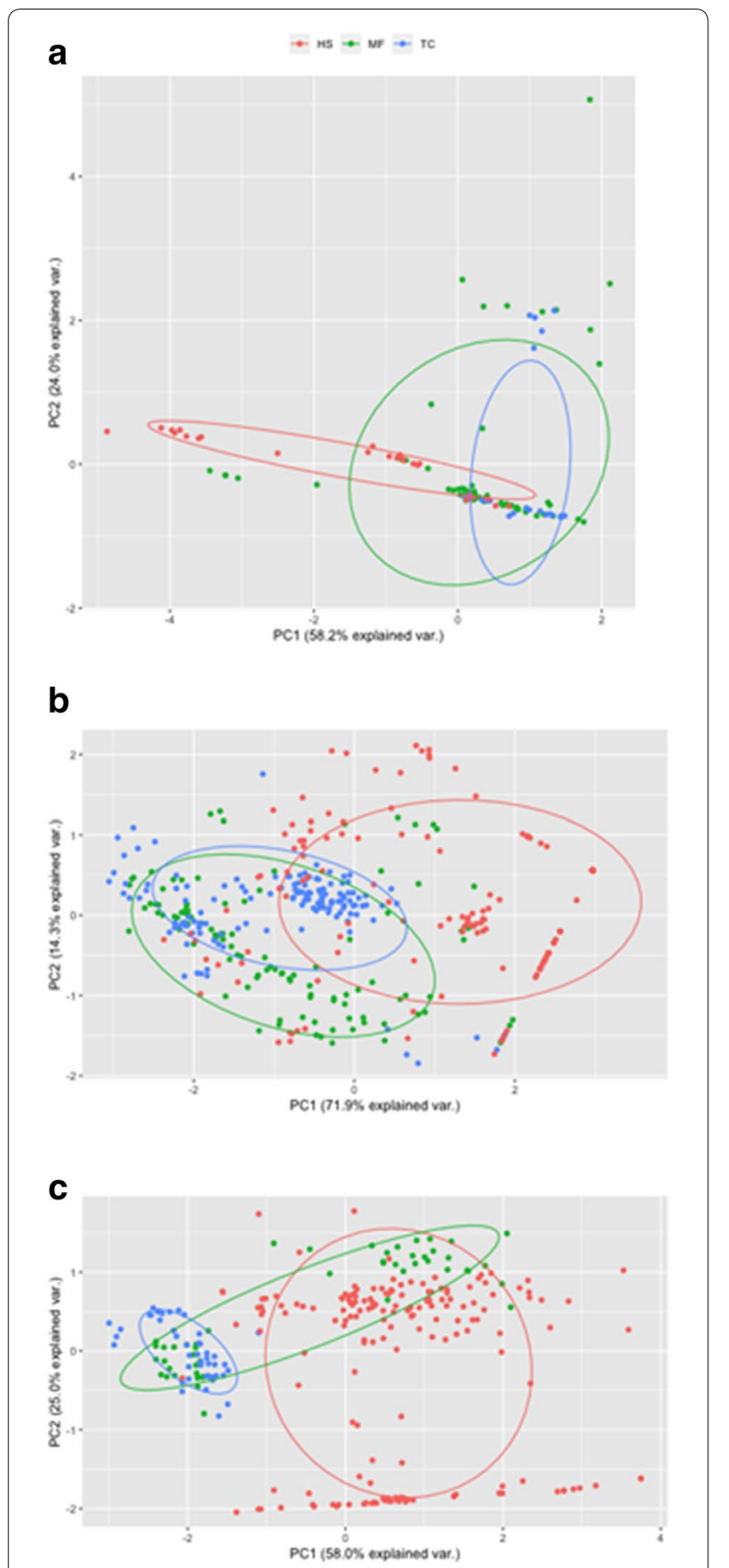

Fig. 3 Principal components analysis of the antibiotic resistance profiles. a MRSA; b Enterococcus spp.; c other Enterobacteriaceae

of Bacteroidetes than HS. In contrast, Acidobacteria was found exclusively in HS but not in the MF nor TC (Fig S2A). In genus level, higher Bacteroides and Prevotella were observed in HS in comparison to the two NHPs (Additional file 2: Fig. S2B).
We showed that the distribution pattern of Operational Taxonomic Unit (OTUs) from the two NHPs were more similar to each other than HS (Fig. 4a). Interestingly however, two samples from MF were clustered together with HS along component PC1. Further investigation into the differentially abundant OTUs revealed higher prevalence of Bacteroides uniformis (OTU00003), Bacteroides caccae (OTU00085), Bifidobacterium longum (OTU00132) in HS in comparison to the two primate species. In contrast, NHPs exhibited higher prevalent of OTUs comprising Christensenellaceae, Ruminococcaceae, Clostridiales, and Oscillospira from the phylum Firmicutes (Fig. 5, Additional file 3: Table S1).

\section{Comparison of predicted functional metagenome between MF, TC and HS}

The distribution of the predicted functional metagenome was illustrated in Fig. 4b. As per the distribution of the bacterial composition, the two NHPs showed higher similarity in predicted functions than HS. Notably, significantly greater abundance of KEGG ortholog related to lipid and carbohydrate metabolism were detected in HS (e.g. K00988 and K06859) (Additional file 4: Table S2). In contrast, the gut community of NHPs harboured more KEGG ortholog for bacterial infection and colonization such as bacterial mobility protein (e.g. K02397, K02413, K02418) and sporulation (K06331) (Fig. 6a, b).

\section{Discussion}

\section{AMR in the studied hosts}

Our findings indicated that isolates from HS has the highest levels of resistance amongst the three hosts. Frequent antibiotic exposure in humans is the most probable cause of antibiotic resistance [13]. We initially postulated that the wild NHPs would exhibit lower levels of antibiotic resistance compared to HS due to limited interaction with humans, as well as absence of antibiotic exposure. Evidence from a previous study showed that NHPs at ecotourism sites might have a higher AMR carriage rate due to constant human presence compared to entirely wild counterparts [14]. The acquisition of antibiotic resistance in wild animals may have a serious consequence on the transmission of antibiotic resistance strains. For instance, the wild animal may act as the carrier of the antibiotic resistance strains. The resistant strains may be further transferred to other members of ecosystems through contact and medium such as soil and water [15].

Macaca facsicularis, an endemic species in Asia was found to harbour bacterial species with higher levels of antibiotic resistance than TC. MF is ubiquitous in Peninsular Malaysia, Sabah and Sarawak. Although the species is generally considered to be wild, MF resides at the fringe of the jungle and are well adapted to interact with 

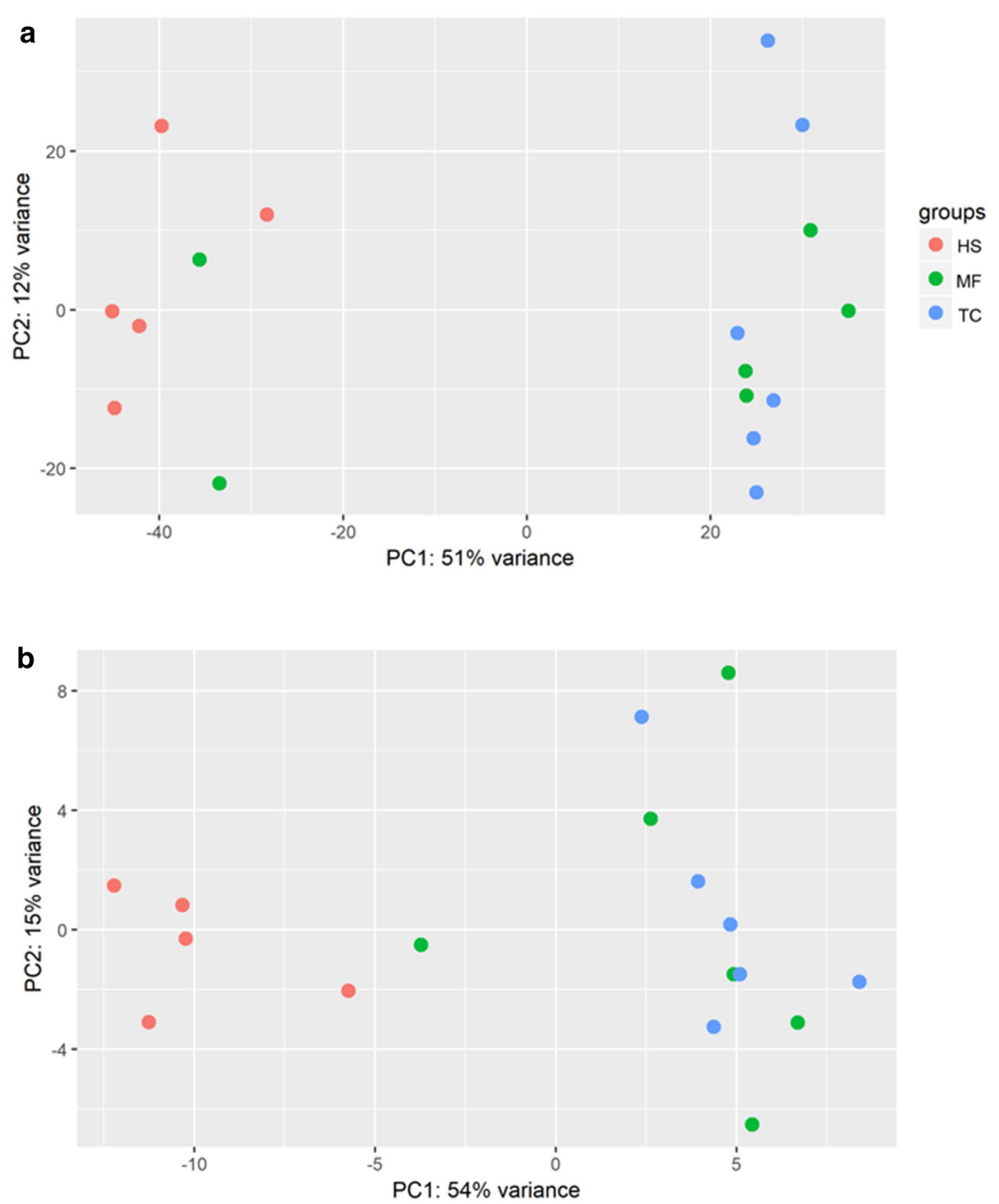

Fig. 4 Euclidean distance-based principle coordinate analysis of regularized transformed matrix of a OTUs; $\mathbf{b}$ predicted functional metagenome

humans (e.g. scavenging food waste). In addition, tourists commonly feed the macaques and this interaction alters the behaviour of animals [16, 17]. Human-animal interactions may also increase the transmission of zoonotic disease to human especially if the subject had been bitten or scratched by the primates $[2,9,16]$. In comparison, TC which are shy and rarely interact with humans [18] showed the lowest carriage rate of antibiotic resistance strains amongst the three hosts studied. Historically,
MF's have been reported to be in closer contact with people and only in recent years have the TC's been monitored to be interacting with people in an urban setting [19].

From our study, Enterococcus species had the highest level of resistance in all hosts, especially for HS. The high levels of resistance to vancomycin for the isolates from the two NHPs is unexplained and require further investigation. A parallel study on black capuchin monkey in 
a

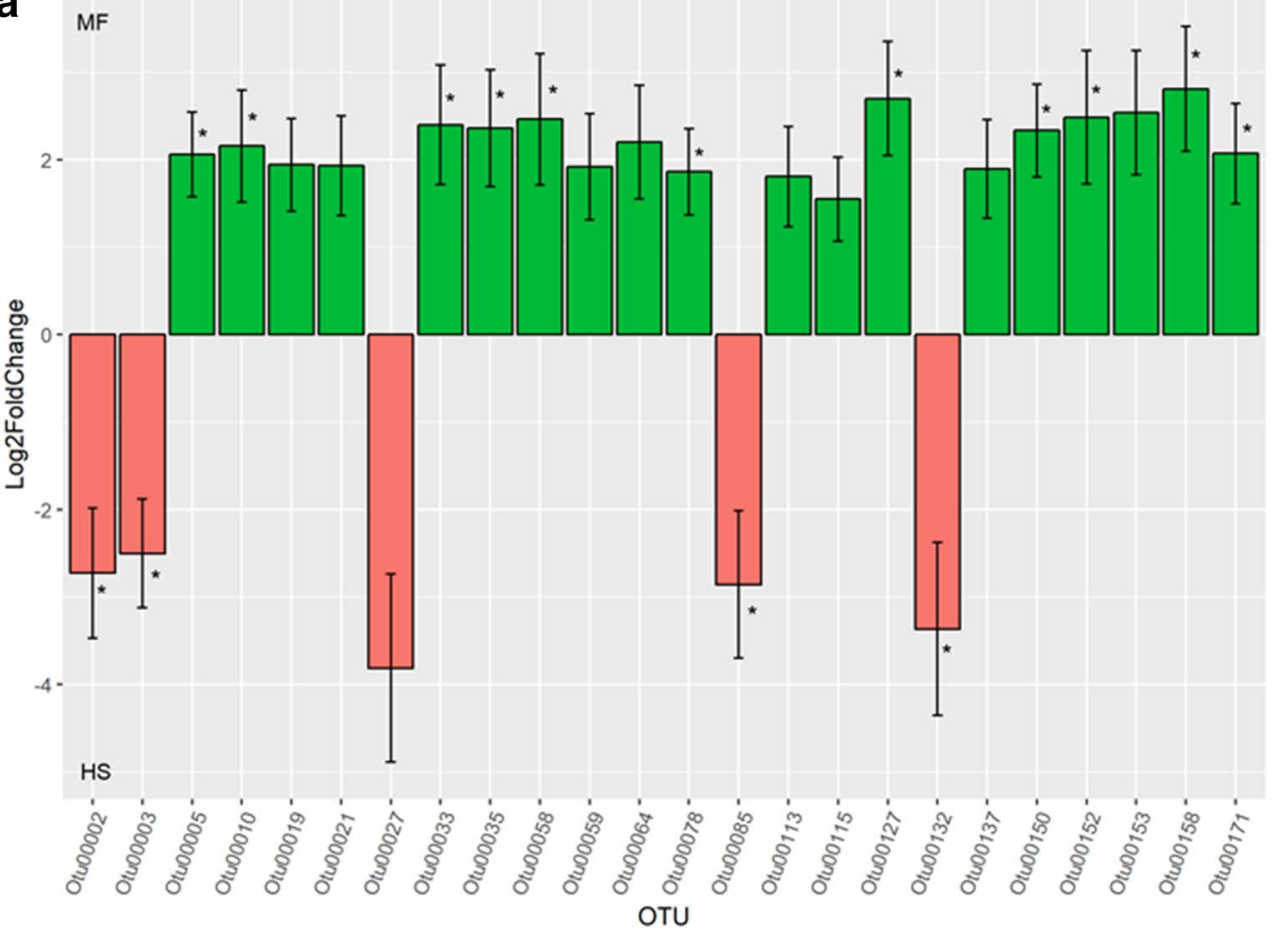

b

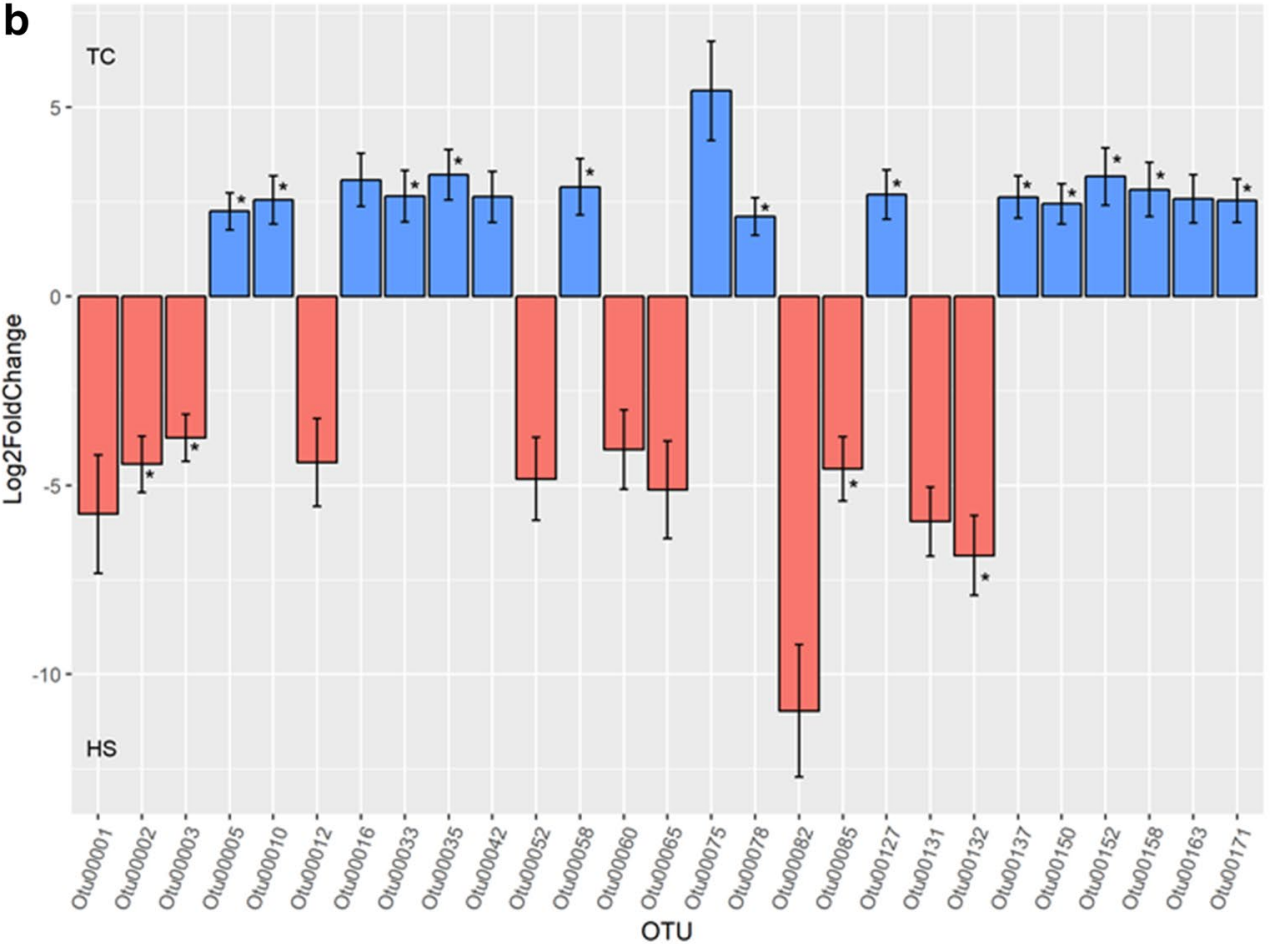

Fig. 5 Significantly abundant OTUs derived using negative log binomial model. a MF vs HS; b TC vs HS

Brazil detected no resistance in the isolated Enterococcus spp. while resistance to other antibiotics including rifampicin, tetracycline, erythromycin, nitrofurantoin, chloramphenicol, and ampicillin were associated with the anthropogenic impact [14]. On the other hand, the Enterobacteriaceae showed highest susceptibility to all 
antibiotics tested. However, this particular finding contract that of Bachiri et al. [20] which showed high prevalence of CTX-M-15 gene in E.coli isolated from Barbary macaque, suggesting wide spread resistance to beta-lactam antibiotic among the wild life in Algeria.

Staphylococcus aureus is $100 \%$ resistant to oxacillin but susceptible to most tested antibiotics. It is interesting to note that resistance to oxacillin is a characteristic of community acquired (CA)-MRSA infection [21]. This might suggest that there is a higher risk of human to primate transfer of resistance $S$. aureus than a zoonotic transfer. In line with this, anthropozoonosis transmission of $S$. aureus was reported from Gambia [22]. Nonetheless, the isolation of unique $S$. aureus ST type in primate suggested that the animal can also be an unappreciated source of MRSA transmission [23].

\section{Host, gut microbiota and AMR carriage rate}

The differences in gut microbiota across the studied hosts coincided with the hosts' diet. Firmicutes and Bacteroidetes are the main dominant phyla in both NHPs and HS. The NHPs in this study both share some similarly to their diet which mainly consists of plants although MFs are omnivorous. Higher abundance of Firmicutes such as Ruminococceae in MF and TC may be associated with higher fiber diet [24]. For instance, genus Oscillospira which is able to degrade a wide range of glycans is affiliated with the plant-based diets in humans [25]. Conversely, HS was found to harbour higher proportion of Bacteroidetes (Fig. 6a). Members of Bacteroides was previously found to be prevalent in animal-based diet due to its' bile-resistant characteristics and the ability to degrade fatty acid into short chain fatty acids (SCFAs) [26]. Interestingly, Bacteroides caccae which has the capacity to digest dietary plant polysaccharides was enriched in HS [27].

Overall, the predicted functional metagenome of HS showed greater representation of KEGG orthologs related to functions such as amino acid and fatty acid degradation, as well as bile acid catalysis. In contrast, the gut microbial community of NHPs exhibited more functions related to bacterial colonization and replication, potentially reflecting the ecological process of functionally diverse environmental bacteria establishing within the animal host [28].

It is noteworthy that the lower bacterial diversity in HS (Additional file 1: Fig. S1) correlated with the higher abundance of antibiotic resistant strains (Fig. 1). In addition, the distribution of antibiotic resistance profile (Fig. 3) is consistent with the composition of gut microbial community and functions (Fig. 4) where higher similarity in MF and TC as opposed to HS was observed. It is recognized that the gut bacterial assemblage may control for the colonization of pathogenic bacteria, including those that are antibiotic resistance [29, 30]. As such, while the lower presentation of antibiotic resistant isolates in the primate may be explained by lower exposure to antibiotic, the native bacterial composition may also play a role in preventing the establishment of the viable colony of the resistant strains in the gut. Furthermore, the gut microbiota may be the reservoir for transfer of antibiotic resistance gene via horizontal gene transfer [31]. As NHPs harbour different gut microbiota in comparison to humans, the transmission of antibiotic resistant pathogens may facilitate transfer of resistance to conventionally susceptible bacterial taxa.

\section{Conclusion}

Activities such as feeding, petting and photography in ecotourism narrows the gap between humans and NHPs, inevitably increasing the potential for the spread of pathogens specifically antibiotic resistant pathogens. In this study, the isolates obtained from the two studied NHPs were found to exhibit lower antibiotic resistance in comparison to the human subject. The results might indicate low rate of transfer from human to primates in the nature park but still a likely possibility. We speculate that the low carriage rate may also be contributed by differences in gut microbial composition, which control the colonization of the resistant pathogens. We argue that provisioning of food to NHPs could alter gut microbiome in due course and over time affect carriage rates. A greater awareness about pathogen transfer to tourists and residents is important to avoid acquiring pathogens but also spreading it which could impact wildlife in the future. We advocate that clear guidelines at tourist sites where unmonitored human-animal interaction takes place should be available to prevent the risk of anthropozoonosis as well as zooanthroponosis.

\section{Methods}

\section{Ethical approval}

Ethics approval for collection of human and non-human primate sampling was obtained from the Perdana University-Internal Review Board (PU-IRB) and was granted under IRB ID: PU IRBHR0088.

\section{Study location}

Kuala Selangor Nature Park (KSNP), and Bukit Melawati in Kuala Selangor, Malaysia were selected as the study sites due to its varied landscape of natural parks as well as popular tourist destination where people interact with wildlife. The ecotourist area is adjacent to a Bukit Melawati residential community equipped with hostels, shops 

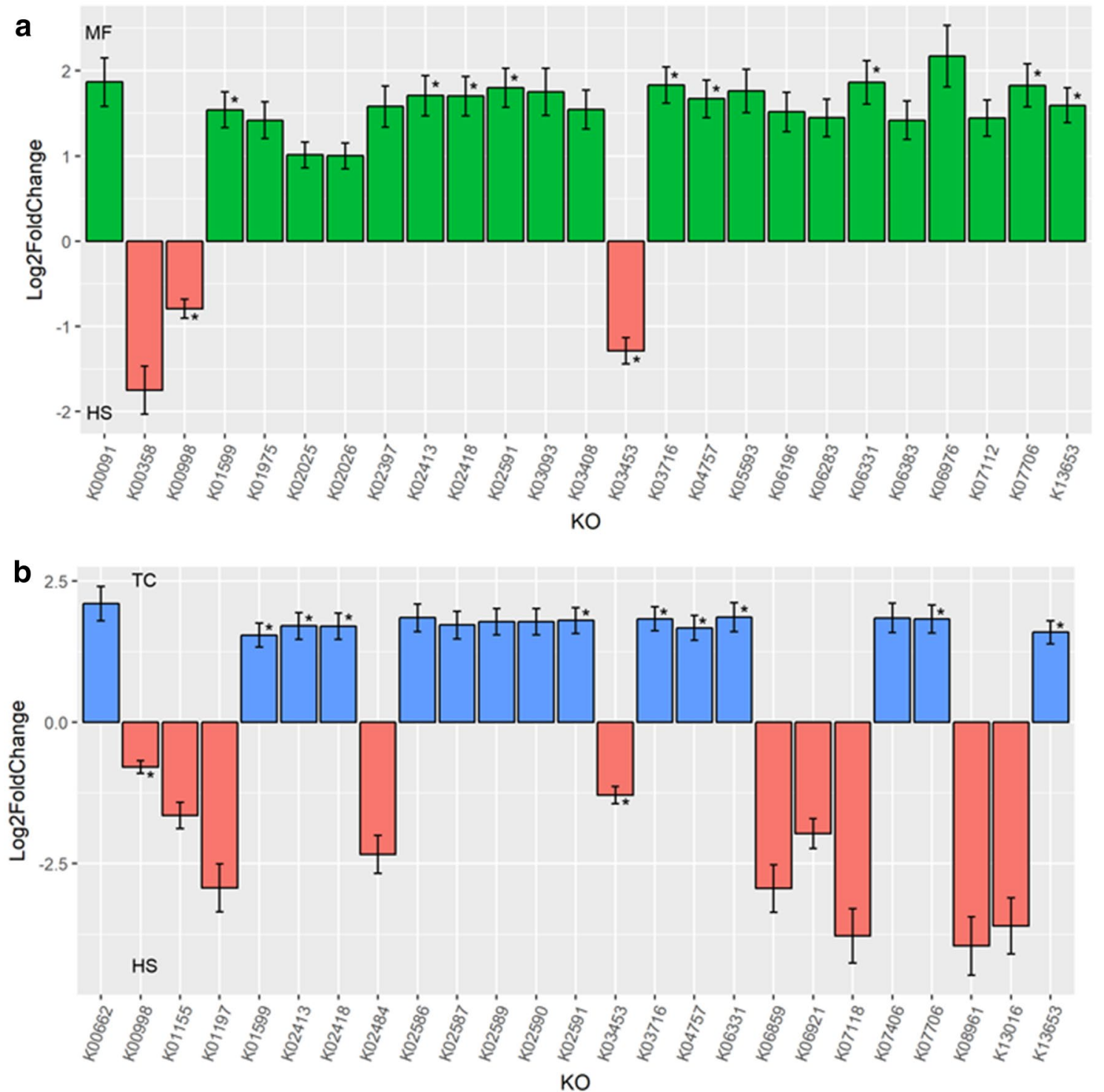

Fig. 6 Significantly abundant KEGG orthologs derived using negative log binomial model. a MF vs HS; b TC vs HS

and schools. The park has been in operation since 1987 and covers over 200 hectares of coastal land, which is mainly mangrove swamps.

\section{Study hosts}

The KSNP is a habitat for a significant number of NHPs, amongst which are long-tailed macaques (Macaca fascicularis) and silver leaf monkeys (Trachypithecus cristatus) (Fig. 7). The non-human primates used in this study. A: Macaca fascicularis (MF), B: Trachypithecus cristatus (TC)). The park was divided into different zones based on the locations where the NHPs were usually found. Zones
$\mathrm{A}, \mathrm{B}, \mathrm{C}$, and $\mathrm{H}$ were the high-interaction zones between NHPs and humans, while the rest (D-K) were mediumand low- interaction zones.

\section{Sample location}

A total of 55 fresh fecal samples were collected, which were made up of 20, 20 and 15 samples of MF, TC and human (HS), respectively. NHPs samples were collected freshly upon defecation and consisted of both male and female samples. MF are omnivorous and TC are predominantly folivorous. However, both species were seen to be provisioned with similar human food like bread, 
a

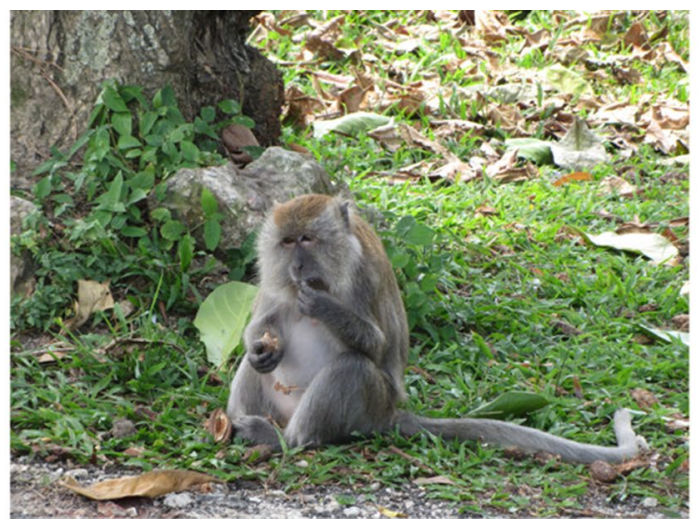

b

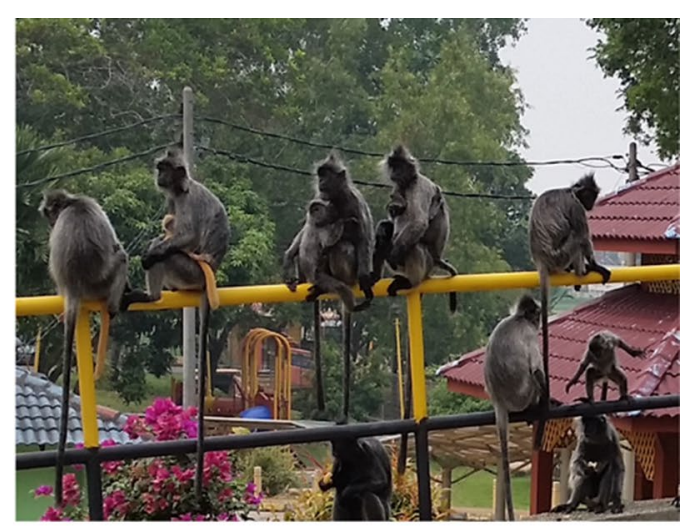

Fig. 7 The non-human primates in the study. a Macaca fascicularis (MF) b Trachypithecus cristatus (TC)

nuts, chips etc. Efforts were made to avoid repeat sampling from the NHPs during the same day. HS samples were collected from healthy volunteers over the age of 18 who were visiting the Ecotourism Park or were residents in the neighbouring area at Kuala Selangor. Four samples were from tourist and the remaining 11 were from residents. Seven of the samples were from people with the age range of 40-60 years old, the remaining 8 were from those between 19 and 28 years old. The type of diet consumed by the HS were not documented. All fecal samples were collected between 10 in the morning and 6 in the evening within a period of 8 months to minimize diurnal variation between February to September 2016. The samples were placed in fecal containers and labeled. The samples were placed in an ice box immediately after collection to preserve bacterial content in the samples. Samples were transported to the lab daily in an ice box and prior to storage, each fecal sample was weighed to $0.1 \mathrm{~g}$ and aliquoted into $1.5 \mathrm{~mL}$ Eppendorf tubes. The samples were then stored at $-20{ }^{\circ} \mathrm{C}$ in the freezer until further use.

\section{Isolation and enumeration of bacteria}

Nine hundred $\mu \mathrm{L}$ of $0.9 \%$ sodium chloride (Kollin, USA) was added to $0.1 \mathrm{~g}$ of the fecal sample which had been aliquoted earlier. The mixture was homogenized using a vortex mixer for $10 \mathrm{~s}$. Dilutions up to $10^{-1}, 10^{-2}, 10^{-3}$, $10^{-4}$ and $10^{-5}$ solution were prepared through ten-fold serial dilutions. One hundred $\mu \mathrm{L}$ of each dilution was then inoculated on Tryptone Soya Agar, TSA (Oxoid, United Kingdom) in triplicates under aseptic condition. The plates were then incubated for $18-24 \mathrm{~h}$ at $37{ }^{\circ} \mathrm{C}$. Approximately 30 to 300 colonies were selected for replica plating.

\section{Staphylococcus spp.}

Sample was plated onto Mannitol Salt Agar, (MSA), (Oxoid, United Kingdom) for the selection of Staphylococcus spp., then the MSA plates were subsequently plated onto ChromMRSA (Chromagar, Oxoid, United Kingdom) for isolation of methicillin-resistant Staphylococcus aureus (MRSA). These plates were incubated for $18-24 \mathrm{~h}$ at $37^{\circ} \mathrm{C}$ before enumeration of the colonies was carried out.

\section{Enterobacteriaceae}

Enterococcus spp. was isolated using Slanetz and Bartley Medium (SBM) (Oxoid, United Kingdom) while the isolation of other Enterobacteriaceae was carried out using Eosin Methylene Blue Agar (EMBA) (Oxoid, United Kingdom). The SBM plates were incubated for $48 \mathrm{~h}$ at $30^{\circ} \mathrm{C}$ and EMB plates were incubated for $18-24 \mathrm{~h}$ at $37^{\circ} \mathrm{C}$. Subsequently, enumeration of the colonies was carried out.

\section{Antibiotic susceptibly test}

Antibiotic susceptibility tests were carried out using agar disc diffusion method using antibiotic impregnated discs (CLSI, 2018). A bacterial lawn was established using a sterile cotton swab whereby the concentration of the bacteria was adjusted according to 0.5 MacFarland $\left(1.5 \times 10^{8}\right.$ Colony forming units $(\mathrm{CFU} / \mathrm{mL})$ Then, discs containing antibiotics were placed onto the agar plates using the incubation condition as described in the method used for bacterial isolations. The diameter of the zone of inhibition was recorded for all antibiotics post-incubation. Distilled water was used as a negative control and the experiment was carried out in triplicates. E. coli ATCC 25922 and S. aureus ATCC 25923 were used as control strains in the disc diffusion test. 
MRSA, Enterococcus spp. and other Enterobacteriaceae isolates from ChromMRSA, SBM and EMBA were tested for antibiotic resistance using specific range of antibiotics (Table 1). For instance, MRSA was tested with oxacillin, tetracycline, cefoxitin, linezolid and vancomycin. For Enterococcus spp., ampicillin, tetracycline, vancomycin, penicillin and linezolid were used. Lastly, ampicillin, gentamicin, tetracycline, cefazolin and ceftazidime were tested on other Enterobacteriaceae. All reference ranges were adapted from CLSI 2018.

Twenty $\mu \mathrm{L}$ of antibiotics with defined concentration were impregnated into the $6 \mathrm{~mm}$ disks (Oxoid, United Kingdom) and classified into resistant, intermediate and susceptible based on CLSI (2018). Putative MRSA, enterococci and other Enterobacteriaceae isolates were subjected to disc diffusion testing using Mueller-Hinton Agar (MHA). Briefly, all isolates were adjusted to 0.5 MacFarland $\left(1.5 \times 10^{8} \mathrm{CFU} / \mathrm{mL}\right)$ before the disks were placed onto MHA. The diameter of inhibition zones for each antibiotic were measured after $24 \mathrm{~h}$ of incubation at $37{ }^{\circ} \mathrm{C}$ for detection of antibiotic resistance in Enterococcus spp. and other Enterobacteriaceae. For detection of MRSA, the plates were incubated for $24 \mathrm{~h}$ at $30^{\circ} \mathrm{C}$ [32].

\section{DNA extraction}

Total genomic DNA from the fecal samples was extracted using the QIAamp stool DNA mini kit according to manufacturer's protocol (Qiagen, Valencia, CA). The extracted DNAs were purified and quantified using Nanodrop (USA) at $260 \mathrm{~nm}$ and $280 \mathrm{~nm}$ prior to $16 \mathrm{~S}$ sequencing using Illumina Miseq.

\section{Amplicon sequencing of $16 \mathrm{~S}$ rDNA gene}

In total, 17 samples were obtained from the three hosts (HS, MF and TC) i.e. six samples for MF and TC and five for HS. All sequences obtained were submitted to GenBank under BioProjectID: PRJNA590002. The taxonomic diversity presented in microbial communities was analysed through sequence variation in the $16 \mathrm{~S}$ ribosomal RNA (rRNA) gene. A total of 2,262,680 raw reads generated from Illumina paired-end sequencing were processed and filtered using Mothur version 1.39.5 [33]. The sequences were clustered and assigned to operational taxonomic unit (OTUs) using the reference SILVA SEED Database Release 132 [34]. Chimeric sequences were identified and removed using VSEARCH, which was implemented within the Mothur pipeline. The final dataset consisted of 669 OTUs from $1,045,777$ sequences, with mean length of $418 \mathrm{bp}$. The data was rarefied to equal depth of 25,273 sequences per sample. Alpha diversity was compared using Shannon's Diversity Index, Simpson's Diversity Index and Pielou's Evenness. In addition, bar charts were constructed using phyloseq package [35] to display the proportional differences in genus and phylum across groups.

Overall differences in bacterial composition between hosts (beta diversity) were evaluated using Permutational Multivariate Analysis of Variance (PERMANOVA). Further, taxa showing significant differences in abundance between host species were identified using negative log binomial model implemented in DeSeq2 R package [36].

\section{Supplementary information}

Supplementary information accompanies this paper at https://doi. org/10.1186/s13099-020-00352-x.

Additional file 1: Fig. S1. Comparison of Alpha Diversity Indices across $\mathrm{MF}, \mathrm{TC}$ and $\mathrm{HS}$.

Additional file 2: Fig. S2. Distribution of A) Phylum-based and B) Genusbased bacterial composition from MF, TC and HS.

Additional file 3: Table S1. The taxonomic identity of significantly different OTUs detected using negative binomial log model.

Additional file 4: Table S2. The identity of significantly different KEGG orthologs detected using negative binomial log model.

\section{Abbreviations}

NHP: Non-human primates; MF: Macaca fascicularis; TC: Trachypithecus cristatus; HS: Homo sapiens; AMR: Antimicrobial resistance; KSNP: Kuala Selangor Nature Park; MRSA: Methicillin-resistant Staphylococcus aureus; CA-MRSA: Community acquired-methicillin-resistant Staphylococcus aureus; AHI: Animal human interaction; CLSI: Clinical \& Laboratory Standards Institute; MSA: Mannitol Salt Agar; SBM: Slanetz and Bartley Medium; EMBA: Eosin Methylene Blue Agar.

\section{Acknowledgements}

The authors wish to thank Fiqri Mustaqqim for helping in sample collection and Perdana University for providing research facilities.

\section{Authors' contributions}

CCW and AAHS drafted the manuscript. AN, TZH and LYL processed the samples. AN performed the data analysis. PSJ and KJ assisted in sample collection and finalising of the manuscript. LSHE developed and designed the experiments and proofread the final manuscript. All authors read and approved the final manuscript.

\section{Funding}

This work is supported by the Malaysian Medical Association Foundation and the Fundamental Research Grant Schemes (FRGS/2014/SKK01/PERDANA/02/1 and FRGS/1/2018/SKK11/PERDANA/02/1).

\section{Availability of data and materials}

The datasets used and/or analysed during the current study are available from the corresponding author on reasonable request. All data generated or analysed during this study are included in this published article (and its additional files).

\section{Ethics approval and consent to participate}

Ethics approval for collection of human and non-human primate sampling were obtained from the Perdana University-Internal Review Board (PU-IRB) and was granted under IRB ID: PU IRBHR0088.

Consent for publication

Not applicable.

Competing interests

The authors declare they have no competing interests. 


\begin{abstract}
Author details
${ }^{1}$ School of Pharmacy, Monash University Malaysia, Jalan Lagoon Selatan, 47500 Bandar Sunway, Selangor Darul Ehsan, Malaysia. ${ }^{2}$ Health Science Division, Abu Dhabi Women's College, Higher Colleges of Technology, 41012 Abu Dhabi, UAE. ${ }^{3}$ Royal College of Surgeons in Ireland, Perdana University, MAEPS Building, 43400 Serdang, Selangor, Malaysia. ${ }^{4}$ Faculty of Health and Medical Sciences, University of Surrey, Guildford GU2 7AL, UK. ${ }^{5}$ Centre for Bioinformatics, School of Data Sciences, Perdana University, MAEPS Building, 43400 Serdang, Selangor, Malaysia. ${ }^{6}$ The Davies Research Centre, School of Animal and Veterinary Sciences, University of Adelaide, Roseworthy, SA 5371, Australia. ${ }^{7}$ Animal Neighbours Project, School of Veterinary Medicine, Faculty of Health and Medical Sciences, University of Surrey, Guildford GU2 7AL, UK.
\end{abstract}

Received: 14 September 2019 Accepted: 28 February 2020

Published online: 10 March 2020

\section{References}

1. Akyol M, Tatoğlu FY, Ustaoğlu M. Financing economic growth by dual banking in Malaysia: Empirical Evidence. In: Ustaoğlu M, INcekara A, editors. Balancing Islamic and conventional banking for economic growth: empirical evidence from emerging economies. Cham: Springer; 2017. p. 69-84.

2. Carne C, Semple S, MacLarnon A, Majolo B, Maréchal L. Implications of tourist-Macaque interactions for disease transmission. EcoHealth. 2017;14(4):704-17.

3. Muehlenbein MP, Martinez LA, Lemke AA, Ambu L, Nathan S, Alsisto S, Sakong R. Unhealthy travelers present challenges to sustainable primate ecotourism. Travel Med Infecti Dis. 2010;8(3):169-75.

4. Lee WH, Moscardo G. Understanding the impact of ecotourism resort experiences on tourists' environmental attitudes and behavioural intentions. J Sustain Tour. 2005;13(6):546-65.

5. Buckley RC, Morrison C, Castley JG. Net effects of ecotourism on threatened species survival. PLoS ONE. 2016;11(2):e0147988.

6. Allen HK, Donato J, Wang HH, Cloud-Hansen KA, Davies J, Handelsman J. Call of the wild: antibiotic resistance genes in natural environments. Nat Rev Microbiol. 2010;8:251.

7. Trinh P, Zaneveld JR, Safranek S, Rabinowitz PM. One health relationships between human, animal, and environmental microbiomes: a mini-review. Front Public Health. 2018;6:235.

8. Ducarmon QR, Zwittink RD, Hornung BVH, van Schaik W, Young VB, Kuijper EJ. Gut microbiota and colonization resistance against bacterial enteric infection. Microbiol Mol Biol Rev. 2019;83(3):e00007-00019.

9. Blancou J, Chomel BB, Belotto A, Meslin FX. Emerging or re-emerging bacterial zoonoses: factors of emergence, surveillance and control. Vet Res. 2005:36(3):507-22.

10. Unwin T, Smith A. Behavioral differences between provisioned and non-provisioned Barbary macaques (Macaca sylvanus). Anthrozoös. 2010;23(2):109-18.

11. McLennan MR, Spagnoletti N, Hockings KJ. The implications of primate behavioral flexibility for sustainable human-primate coexistence in anthropogenic habitats. Int J Primatol. 2017;38(2):105-21.

12. Maréchal L, MacLarnon A, Majolo B, Semple S. Primates' behavioural responses to tourists: evidence for a trade-off between potential risks and benefits. Sci Rep. 2016:6:32465.

13. Rousham EK, Unicomb L, Islam MA. Human, animal and environmental contributors to antibiotic resistance in low-resource settings: integrating behavioural, epidemiological and one health approaches. Proc Biol Sci. 1876;2018(285):20180332.

14. Grassotti TT, de Angelis Zvoboda D, da Fontoura Xavier Costa L, de Araújo AJG, Pereira RI, Soares RO, Wagner PGC, Frazzon J, Frazzon APG. Antimicrobial resistance profiles in Enterococcus spp. isolates from fecal samples of wild and captive black Capuchin monkeys (Sapajus nigritus) in South Brazil. Front Microbiol. 2018;9:2366

15. Vittecoq M, Godreuil S, Prugnolle F, Durand P, Brazier L, Renaud N, Arnal A, Aberkane S, Jean-Pierre H, Gauthier-Clerc M, et al. Antimicrobial resistance in wildlife. J Appl Ecol. 2016;53(2):519-29.

16. Fuentes A. Human culture and monkey behavior: assessing the contexts of potential pathogen transmission between macaques and humans. Am J Primatol. 2006:68(9):880-96.
17. Soulsbury CD, White PCL. Human-wildlife interactions in urban areas: a review of conflicts, benefits and opportunities. Wildl Res. 2015;42(7):541-53.

18. Harding LE. Trachypithecus cristatus (Primates: Cercopithecidae). Mammal Species. 2010;42(1):149-65.

19. Atiqah Tahir N, Ismail A, Rahman F. Daily activity budget of silver leaf monkeys (Trachypithcus cristatus) in Kuala Selangor Nature Park (KSNP), Peninsular Malaysia Malay. Nature J. 2017:69:337-43.

20. Bachiri T, Bakour S, Ladjouzi R, Thongpan L, Rolain JM, Touati A. High rates of CTX-M-15-producing Escherichia coli and Klebsiella pneumoniae in wild boars and Barbary macaques in Algeria. J Glob Antimicrobia Resist. 2017:8:35-40.

21. Gonzalez BE, Martinez-Aguilar G, Hulten KG, Hammerman WA, Coss-Bu J, Avalos-Mishaan A, Mason EO Jr, Kaplan SL. Severe Staphylococcal sepsis in adolescents in the era of community-acquired methicillin-resistant Staphylococcus aureus. Pediatrics. 2005;115(3):642-8.

22. Senghore M, Bayliss SC, Kwambana-Adams BA, Foster-Nyarko E, Manneh J, Dione M, Badji H, Ebruke C, Doughty EL, Thorpe HA, et al. Transmission of Staphylococcus aureus from humans to green monkeys in the Gambia as revealed by whole-genome sequencing. Appl Environ Microbiol. 2016:82(19):5910.

23. Soge OO, No D, Michael KE, Dankoff J, Lane J, Vogel K, Smedley J, Roberts MC. Transmission of MDR MRSA between primates, their environment and personnel at a United States primate centre. J Antimicrob Chemother 2016:71(10):2798-803.

24. Menni C, Jackson MA, Pallister T, Steves CJ, Spector TD, Valdes AM. Gut microbiome diversity and high-fibre intake are related to lower long-term weight gain. Int J Obes. 2017:41:1099.

25. KonikoffT, Gophna U. Oscillospira: a Central, Enigmatic Component of the Human Gut Microbiota. Trends Microbiol. 2016;24(7):523-4.

26. David LA, Maurice CF, Carmody RN, Gootenberg DB, Button JE, Wolfe BE, Ling AV, Devlin AS, Varma Y, Fischbach MA, et al. Diet rapidly and reproducibly alters the human gut microbiome. Nature. 2013;505:559.

27. Sonnenburg ED, Zheng H, Joglekar P, Higginbottom SK, Firbank SJ, Bolam DN, Sonnenburg JL. Specificity of polysaccharide use in intestinal bacteroides species determines diet-induced microbiota alterations. Cell. 2010;141(7):1241-52.

28. McFall-Ngai M, Hadfield MG, Bosch TCG, Carey HV, Domazet-Lošo T, Douglas AE, Dubilier N, Eberl G, Fukami T, Gilbert SF, et al. Animals in a bacterial world, a new imperative for the life sciences. Proc Natl Acad Sci. 2013;110(9):3229-366.

29. Buffie CG, Pamer EG. Microbiota-mediated colonization resistance against intestinal pathogens. Nat Rev Immunol. 2013:13:790.

30. Bahrndorff S, Alemu T, Alemneh T, Lund Nielsen J. The microbiome of animals: implications for conservation biology. Int J Genomics. 2016;2016:7.

31. van Schaik W. The human gut resistome. Philos Trans R Soc Lond B Biol Sci. 2015;370(1670):20140087-20140087

32. Brown DF. Detection of methicillin/oxacillin resistance in Staphylococci. J Antimicrob Chemother. 2001;48(Suppl 1):65-70.

33. Schloss PD, Westcott SL, Ryabin T, Hall JR, Hartmann M, Hollister EB, Lesniewski RA, Oakley BB, Parks DH, Robinson CJ, et al. Introducing mothur: open-source, platform-independent, community-supported software for describing and comparing microbial communities. App Environ Microbiol. 2009:75(23):7537.

34. Quast C, Pruesse E, Yilmaz P, Gerken J, Schweer T, Yarza P, Peplies J, Glöckner FO. The SILVA ribosomal RNA gene database project: improved data processing and web-based tools. Nucleic Acids Res. 2013;41(Database):D590-D596596.

35. McMurdie PJ, Holmes S. phyloseq: an R package for reproducible interactive analysis and graphics of microbiome census data. PLOS ONE. 2013;8(4):e61217.

36. Love MI, Huber W, Anders S. Moderated estimation of fold change and dispersion for RNA-seq data with DESeq2. Genome Biol. 2014;15(12):550.

\section{Publisher's Note}

Springer Nature remains neutral with regard to jurisdictional claims in published maps and institutional affiliations. 